PAPER

\title{
High-frequency stability oscillator for surface acoustic wave gas sensor
}

\author{
Shitang $\mathrm{He}^{1, *}$, Wen Wang ${ }^{1}$, Shunzhou $\mathrm{Li}^{1}$, Yong $\mathrm{Pan}^{2}$ and Minghua Liu ${ }^{1}$ \\ ${ }^{1}$ Institute of Acoustics, Chinese Academy of Sciences, \\ No. 21 North 4th Ring Road West, Beijing, 100190 P.R. China \\ ${ }^{2}$ Research Institute of Chemical Defense, Beijing, 102205 P.R. China
}

( Received 30 September 2007, Accepted for publication 5 February 2008)

\begin{abstract}
We present a $300 \mathrm{MHz}$ surface acoustic wave (SAW) oscillator for gas sensor application. As the oscillator element, SAW delay lines on an ST-X quartz substrate with low insertion loss and single-mode-selection capability were developed. And they were structured by Electrode Width Control Single Phase Unidirectional Transducer (EWC/SPUDT) configuration and comb transducer. The coupling of modes (COM) model was used to predict device performance prior to fabrication. The measured frequency response $S_{12}$ showed a good agreement with simulated results; a low insertion loss of less than $9 \mathrm{~dB}$ and a linear phase in the $3 \mathrm{~dB}$ bandwidth were observed. The experimental results show that the baseline noise of the fabricated oscillators was typically up to $\sim 0.7 \times 10^{-7}$ in a laboratory environment with temperature control. The oscillator was successfully applied to a gas sensor coated with fluoroalcoholpolysiloxane (SXFA) as the sensor material for O-isopropyl methyphosphonofluoridate (GB) detection, and a superior threshold detection limit was obtained $\left(\ll 0.4 \mathrm{mg} / \mathrm{m}^{3}\right)$.
\end{abstract}

Keywords: Delay line, Frequency stability, Gas sensor, Oscillator, Surface acoustic wave, Sensitivity

PACS number: 43.60.Lg, 43.66.Jh [doi:10.1250/ast.30.7]

\section{INTRODUCTION}

Gas sensors based on surface acoustic wave (SAW) oscillators structured by delay lines have been the focus of growing interest since Wohltjen and Ressy reported the first studies in 1979, owing to their simplicity, high sensitivity, fast response, low cost and compatibility with IC fabrication [1]. Figure 1 shows the schematic of a typical SAW gas sensor system consisting of a dual delay line oscillator and a sensitive coating deposited between the input and output interdigital transducers (IDTs) of one delay line. The adsorption of a specific gas by the sensitive coating modulates the phase velocity of the acoustic wave, producing changes at the output of the SAW device, which can be read out by recording the frequency of the oscillator. As the sensor element, the frequency stability of the SAW oscillator directly affects the threshold detection level $(\Delta m)$ and sensitivity according to

$$
\Delta f / f_{0}=\left(k_{1}+k_{2}\right) \times f_{0} \times \Delta m / s,
$$

where $\Delta f / f_{0}$ is the relative change of the oscillator frequency, $k_{1}$, and $k_{2}$ are material constants of the substrate, $f_{0}$ the operating frequency, and $s$ the area of

*e-mail: heshitang@mail.ioa.ac.cn the sensitive film. Moreover, a further increase in operating frequency will result in a large increase in the sensitivity of the sensor (Eq. (1)).

It is known that the insertion loss of the SAW device and noise of the oscillator circuit are the main factors that influence the frequency stability of the oscillator [2]. Many groups have reported some successful SAW oscillators of different design for gas sensing. However, the current oscillator used for gas sensing suffers from high insertion loss of the oscillator element (delay line: $15-40 \mathrm{~dB}$ depending on different operating frequency) resulting from the bidirectional IDT structure and quartz piezoelectric substrate with weak electromechanical factors $(0.11 \%$ for ST-X quartz) which is used widely as the oscillator substrate owing to its superior temperature stability (TCD: $\sim 0$ ). Thus the amplifier must be operated in a high gain state which leads to high phase noise, and hence deterioration of the frequency stability. In addition, mode selection in the oscillator is accomplished with the accessional circuit (for example, auto gain control (AGC) system) [2], which induces an increase in oscillator power consumption and degrades the frequency stability of the oscillator. Also, low operation frequency (operation frequency of the most current SAW sensors is around $100 \mathrm{MHz}$ ) obstructs the improvement of the sensitivity of the sensor. 


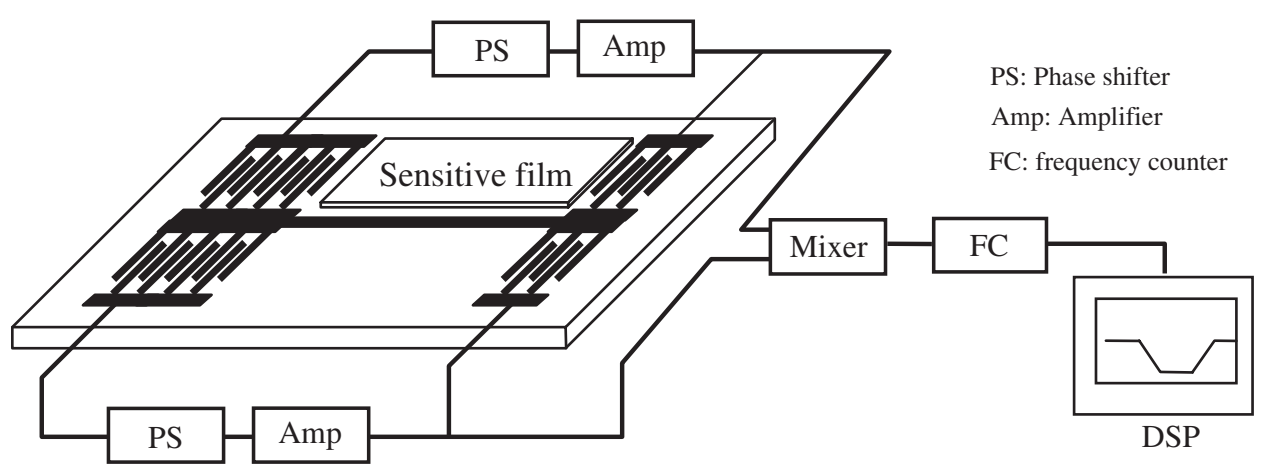

Fig. 1 Schematic of SAW-based sensor system with dual delay line oscillator.

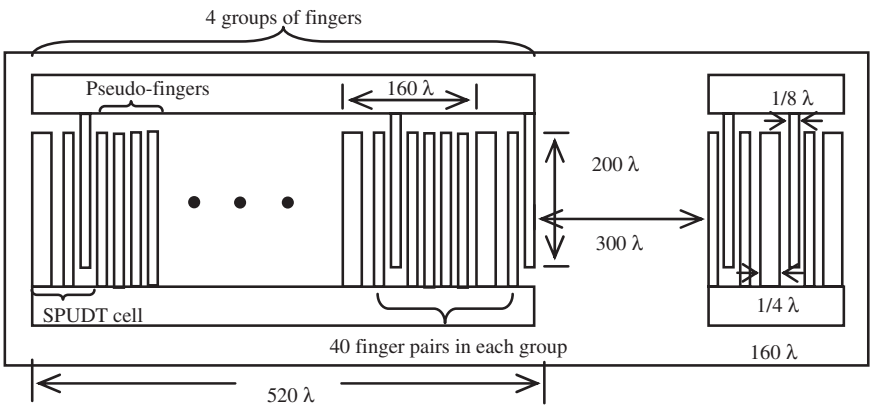

(a)

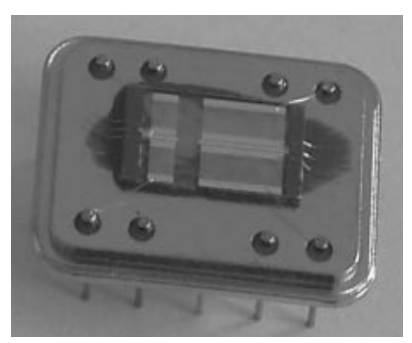

(b)

Fig. 2 (a) SAW delay line with combed and SPUDT structure. (b) Fabricated SAW delay line.

The first purpose of this study is to provide deeper insight into the frequency stability of the SAW oscillator for gas sensing, which is constructed of novel $300 \mathrm{MHz}$ SAW delay lines on an ST-X quartz substrate with low insertion loss (less than $9 \mathrm{~dB}$ ) and single-mode-selection capability, as shown in Fig. 2. High operating frequency makes it possible to improve the sensitivity of the sensor system. Low insertion loss is achieved as a result of the EWC/SPUDT configuration, as shown in Fig. 2(a), which enhances the generated signal in the forward direction but reduces the signal in the reverse direction [3]. Singlemode-selection can be simply accomplished by a comb transducer, which combines the frequency selectivity of two IDTs to suppress all unwanted frequencies, as shown in Fig. 2(a) [2]. In order to maintain the uniformity of acoustic velocity, many pseudofingers are distributed between the teeth of the comb transducer. To predict the device performance, coupling of modes (COM) simulation was performed prior to fabrication. The experimental results showed that, in a laboratory environment with temperature control, superior short-term frequency stability (1h) of the fabricated oscillator was observed.

The other aim of this study is to develop a gas sensor incorporating the fabricated oscillator coated with SXFA as the sensor material for O-isopropyl methyphosphonofluoridate (GB), which provides high sensitivity and selectivity for organic nerve vapor detection. The SXFA coating was prepared by solvent evaporating technology, in which toluene was used as the solvent. The experimental results confirmed the satisfactory operation of the sensor.

\section{COUPLING OF MODES (COM) SIMULATION}

COM modeling is a very efficient technique for the analysis of the SAW device in which small distributed internal reflections are important, such as in the SPUDT structure [4]. Wright deduced the COM equation for SAW devices with EWC/SPUDT; the equation deals with acoustic waves propagating in the forward $(R)$ and reverse directions $(S)$ and incorporates their coupling interaction [5]. The $3 \times 3 P$-matrix representation is used to present the solutions to the COM equations. Based on the COM modeling, the SAW delay line with the SPUDT and comb transducer structure (Fig. 2(a)) was analyzed as follows. Using the zero transducer factors, zero static capacitor and zero reflection coefficients, the $P$ matrix of the pseudofingers distributed in the comb transducer can be deduced. Referring to the cascading relationships [4], the $P$ matrix for all the individual IDT segments in each group was cascaded and described as $P_{\mathrm{IDTi}}(i=1,2, \ldots$, represent the group number). The $P$ matrix for the pseudofingers was also cascaded as $P_{\text {psui }}$. The $P$ matrix for the left transducer 


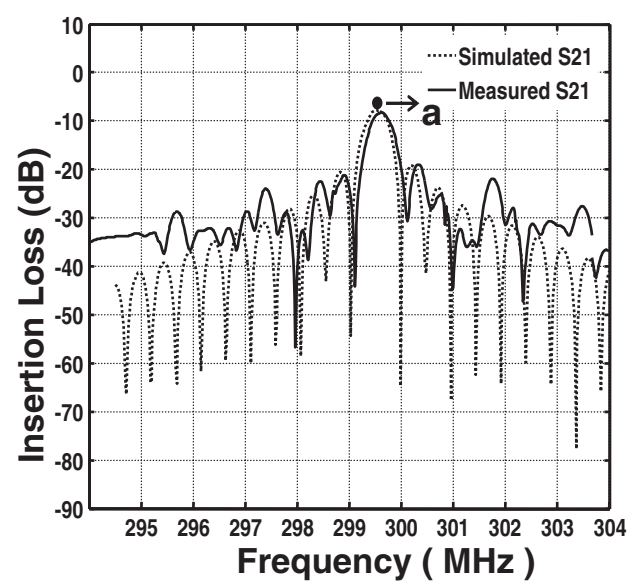

(a)

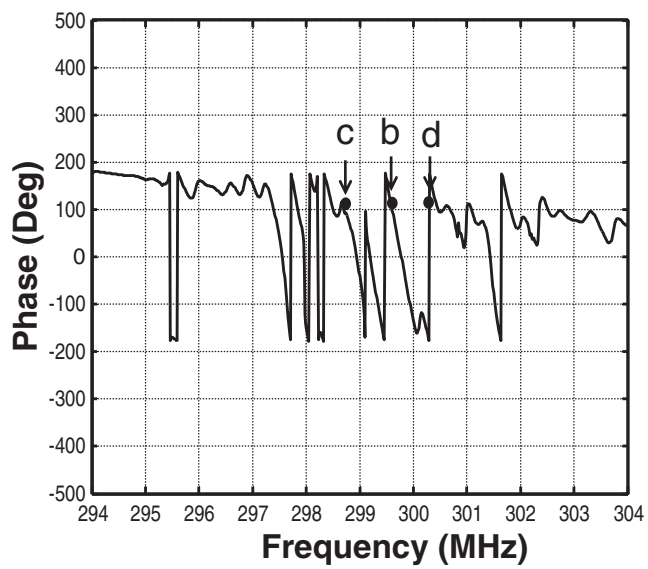

(b)

Fig. 3 Frequency responses of the $300 \mathrm{MHz}$ SAW delay line. (a) Simulated and measured amplitude response. (b) Measured phase response.

(shown in Fig. 2(a)) can be deduced (PL) by cascading between $P_{\mathrm{IDTi}}$ and $P_{\mathrm{psui}}$, while the $P$ matrix for the right transducer in Fig. 2(a) can be described as PR. Therefore, the admittance matrix for the total device was expressed by

$$
Y=\left[\begin{array}{ll}
Y_{11} & Y_{12} \\
Y_{21} & Y_{22}
\end{array}\right]
$$

where

$$
\left\{\begin{array}{l}
Y_{11}=P L_{33}+\frac{P R_{11} P L_{32} P L_{23}}{1-P R_{11} P L_{22}}, Y_{12}=\frac{P R_{13} P L_{32}}{1-P R_{11} P L_{22}} \\
Y_{21}=\frac{P R_{31} P L_{23}}{1-P R_{11} P L_{22}}, Y_{22}=P R_{33}+\frac{P L_{22} P R_{13} P R_{31}}{1-P R_{11} P L_{22}}
\end{array}\right.
$$

Using the admittance matrix solution, the frequency/phase response can be deduced by

$$
\left\{\begin{array}{l}
S_{21}=\frac{-2 Y_{21} \sqrt{G_{\text {in }} G_{\text {out }}}}{\left(G_{\text {in }}+j B_{1}+Y_{11}\right)\left(G_{\text {out }}+j B_{2}+Y_{22}\right)-Y_{12} Y_{21}}, \\
P_{21}=\operatorname{atan}\left(\operatorname{Im}\left(S_{21}\right) / \operatorname{Re}\left(S_{21}\right)\right) \times 180 / \pi
\end{array}\right.
$$

where $G_{\text {in }}$ and $G_{\text {out }}$ are the input and output resistances, respectively of the peripheral circuits, and $B_{1}$ and $B_{2}$ are the susceptances for impedance matching.

\section{TECHNICAL REALIZATION}

\subsection{SAW Delay Line}

A $300 \mathrm{MHz}$ SAW delay line with the EWC/SPUDT and comb transducer structure with an $\mathrm{Al}$ electrode was fabricated by the standard photolithographic techniques. The length of the long transducer was set to $520 \lambda$ with four groups, and was about $80 \%$ of the center-to-center distance between the input and output transducers. The distance between the transducers was $300 \lambda$. In order to limit the total number of $\mathrm{Al}$ fingers in each transducer to about 160, the long transducer was thinned into a comb structure. A large aperture of $\sim 2 \mathrm{~mm}(200 \lambda)$ was used to reduce the acoustic wave diffraction and provide enough area for sensitive film deposition. ST-X quartz was used as the substrate owing to its superior temperature stability. A photograph of the fabricated dual delay line is shown in Fig. 2(b).

Using the HP 8753D network analyzer, the amplitude and phase response of the SAW delay lines were measured under a matched condition, as shown in Figs. 3(a) and (b), respectively. Lower insertion loss $(8.5 \mathrm{~dB})$ and linear phase change in $3 \mathrm{~dB}$ frequency bandwidth were observed, and the measured result was in good agreement with the simulated one (Fig. 3(a)). It also showed the principal of mode selection from the phase response (Fig. 3(b)). We assume only the center frequency of the delay line ( $a$ in Fig. 3(a)) satisfies the oscillator condition, and the corresponding phase is described as $b$. if there is a shift of $2 \pi$ for the phase change (c or $d$ in Fig. 3(b)), even though the phase still meets the oscillator condition, the corresponding insertion loss increases to over $10 \mathrm{~dB}$. Obviously, it is impossible to satisfy the oscillation gain condition [2]. Thus the oscillator works at a single frequency.

\subsection{SAW Oscillator}

The output and input transducers of the SAW delay line were connected by an oscillator circuit made of discrete elements (amplifier with a gain of $25 \mathrm{~dB}$, phase shifter, mixer and LPF, and so on) connected to each other via SMA connectors, as shown in Fig. 4. The output of the amplifier was mixed in order to obtain a difference frequency in the $\mathrm{MHz}$ range. This technique allows us to reduce the influence of the thermal expansion of the substrate and to use simple low-frequency counters. The output of the oscillator was connected to a programmable frequency counter that can be used for an analog indicator or a chart recorder. 


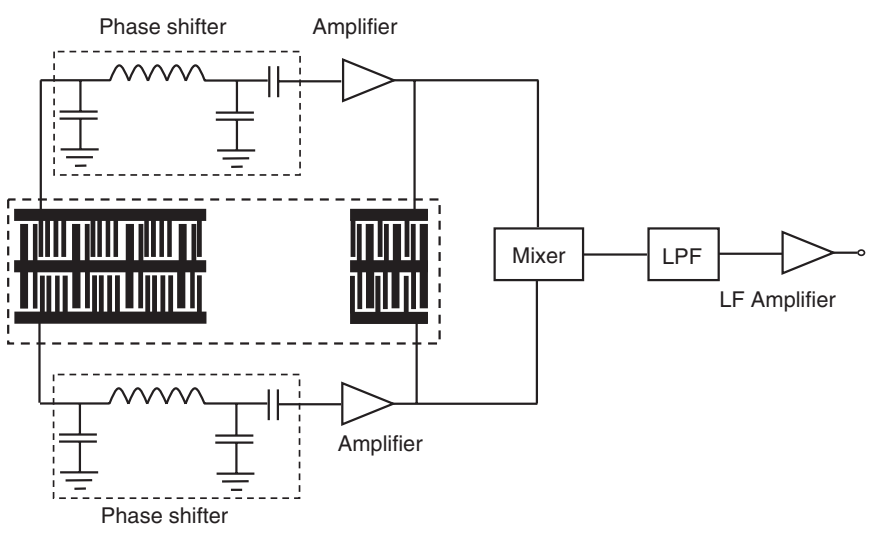

Fig. 4 Oscillator circuit configuration.

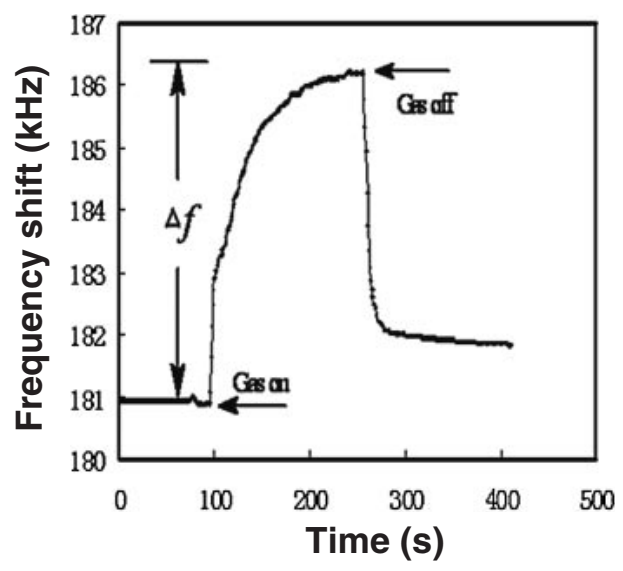

(a)

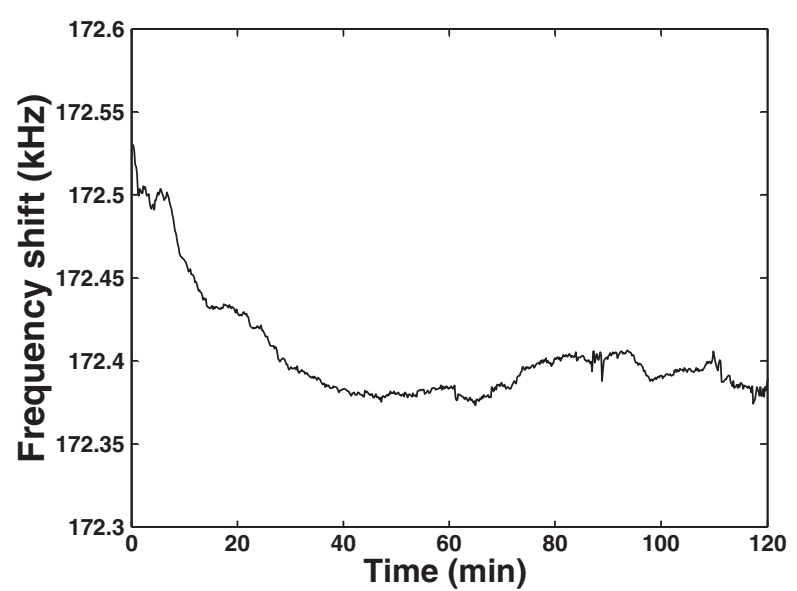

Fig. 5 Typical frequency stability of the new SAW oscillator.

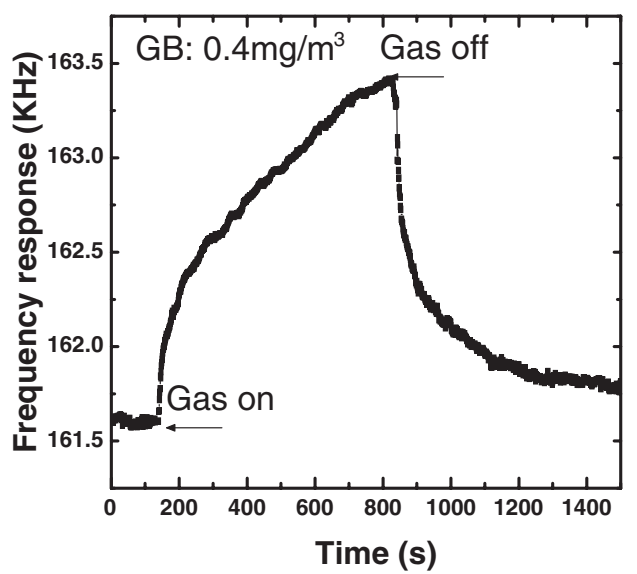

(b)

Fig. 6 Sensor responses to various GB concentrations: (a) $3.4 \mathrm{mg} / \mathrm{m}^{3}$ and (b) $0.4 \mathrm{mg} / \mathrm{m}^{3}$ at $35 \% \mathrm{RH}, 14^{\circ} \mathrm{C}$ and gas flow speed of $1 \mathrm{l} / \mathrm{min}$.

Because of the effect of the oscillator circuit system temperature, a special temperature control was used as a cooling step of the oscillator circuit system to improve the frequency stability of the oscillator [6]. The measured typical short-term frequency stability attained $\sim 0.07 \mathrm{ppm}$ $(20 \mathrm{~Hz})$ in the stable state (Fig. 5).

\subsection{Sensitive Coating Deposition Processes}

In this paper, the solvent-evaporating method was used for the SXFA deposition, owing to its simple operation and low cost. The effectiveness of the solvent evaporating method to produce a stable polymer coating depends on the selection of the solvent type. In our experiment, toluene was used as the solvent.

Before the deposition of the SXFA film on the SAW device surface, the quartz surface was cleaned of any contaminants by a routine cleaning procedure involving rinsing in Piranha solution $\left(\mathrm{V}\left(\mathrm{H}_{2} \mathrm{SO}_{4}\right): \mathrm{V}\left(\mathrm{H}_{2} \mathrm{O}_{2}\right)=3: 1\right)$, a DI water rinse and drying by $\mathrm{N}_{2}$. Then, a $0.1 \mu$ l solution of $0.8 \mathrm{~g} / \mathrm{l} \mathrm{SXFA} /$ toluene was deposited on the cleaned quartz surface for 10 consecutive times. To monitor the film deposition, the sensor response for each time was recorded by the frequency counter (Proteck C3100, Korea). Making use of the law of Sauerbreg, the SXFA thickness was evaluated to be $15 \mathrm{~nm}$.

\section{SENSOR EXPERIMENTS}

\subsection{GB Adsorption}

Under a high GB concentration of $3.4 \mathrm{mg} / \mathrm{m}^{3}$, the vapor test of the fabricated sensor was performed. The output of the oscillator was monitored with the frequency counter under the conditions of $14^{\circ} \mathrm{C}, 35 \% \mathrm{RH}$ and gas flow speed of $1 \mathrm{l} / \mathrm{min}$. Figure 6(a) shows the testing result. Here, the frequency response was recorded every $2 \mathrm{~s}$ so that one point on the graph corresponds to a $2 \mathrm{~s}$ interval. The sensor response showed a rapid rise upon exposure to GB and reaches approximately $40.4 \%$ and $80 \%$ of the equilibrium (saturation) value in $10 \mathrm{~s}$ and $50 \mathrm{~s}$, respectively. When the gas was removed by toluene, the sensor response returned to $\sim 70 \%$ of its initial baseline value within $10 \mathrm{~s}$. The 


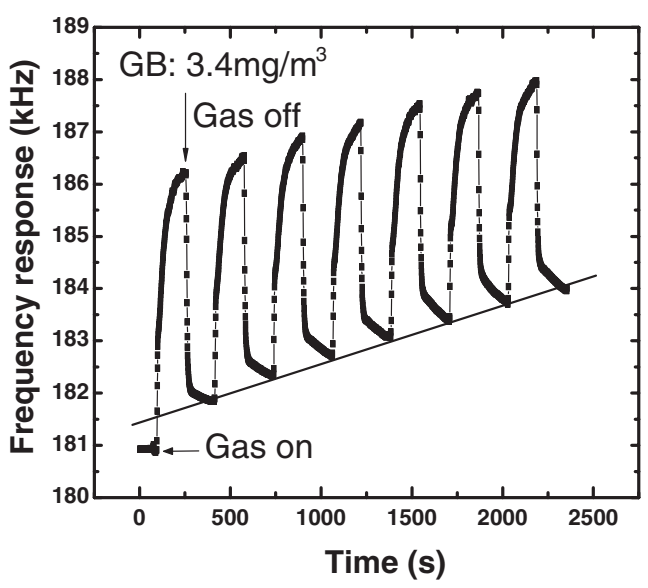

Fig. 7 Sensor response profiles obtained from 7 consecutive 320 -s on-off exposures to $3.4 \mathrm{mg} / \mathrm{m}^{3}$ of GB.

transition of initial state-adsorption-stable equilibration state-desorption-recovery to initial state was clearly observed, and it was also obtained by GB detection with a low concentration of $0.4 \mathrm{mg} / \mathrm{m}^{3}$, in which the sensor provided a signal of almost $1,738 \mathrm{~Hz}$ for $0.4 \mathrm{mg} / \mathrm{m}^{3}$ of GB (Fig. 6(b)). According to the International Union of Pure and Applied Chemistry (IUPAC), detection limits are evaluated as the lowest concentration of an analyte giving a signal of three-times the short-term frequency stability of the sensor system. In our sensor, it maybe possible to improve the detection limit (up to $0.02 \mathrm{mg} / \mathrm{m}^{3}(0.1 \mathrm{ppm})$ ) owing to the high frequency stability of the system $(0.07 \mathrm{ppm})$, which provides a signal-to-noise ratio of more than 25:1.

\subsection{Repeatability}

Response and recovery times to GB gas were tested. SAW frequency response measurements were performed from seven consecutive 320-second on-off exposures to GB at a concentration of $3.4 \mathrm{mg} / \mathrm{m}^{3}$ at $14^{\circ} \mathrm{C}$ and $35 \% \mathrm{RH}$, as shown in Fig. 7. When the gas was infused (on state) into the chamber for $160 \mathrm{~s}$, the frequency response showed a rapid rise and finally reached to its saturation value. When the gas was in the off state, the frequency rapidly fell to its initial value. However, because the absorbed GB is difficult to remove from the SXFA film completely in a short time, GB accumulated in the SXFA film during the periodically repeated measurement, inducing a baseline frequency shift, as shown in Fig. 7, and the phenomenon may be eliminated by extending periodic repetition time. From this promising result, we hypothesize that this sensor has excellent shortterm repeatability in response to GB.

\subsection{Sensitivity Evaluation}

We exposed the sensor to various GB concentrations to evaluate its sensitivity. Figure 8 shows the obtained frequency shifts at various GB concentrations under fixed

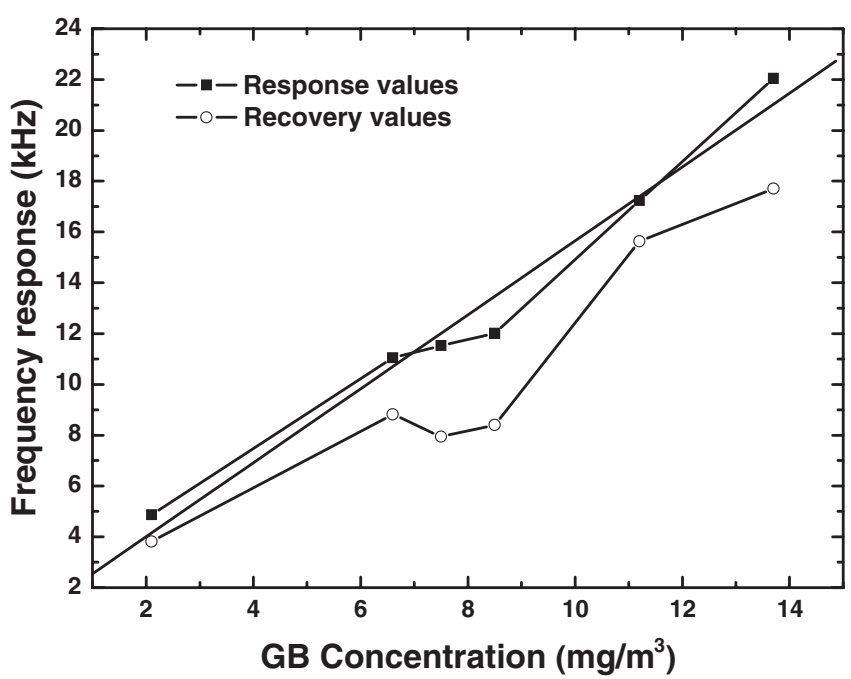

(a)

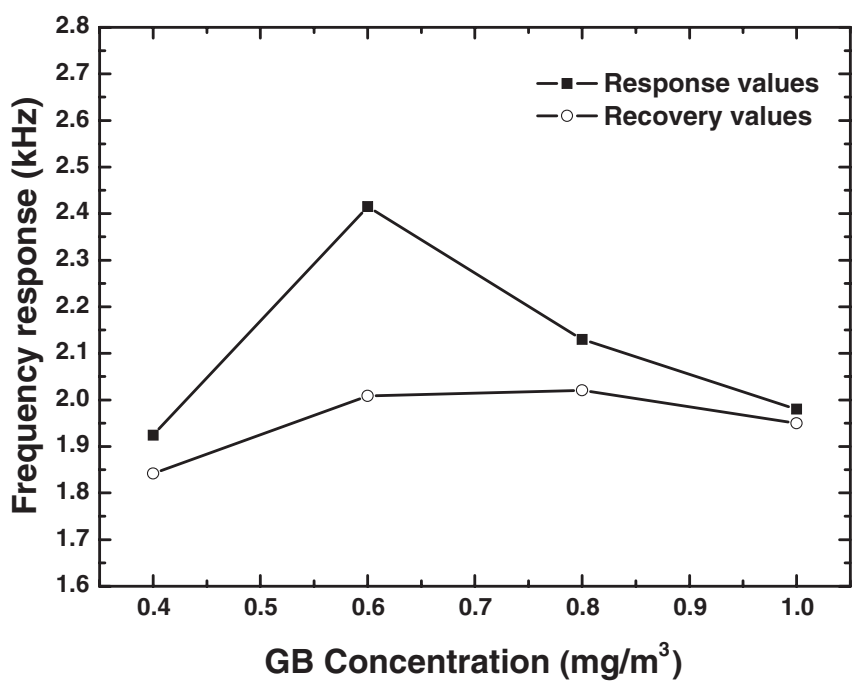

(b)

Fig. 8 Sensor response in terms of GB concentration change at (a) for high GB and (b) low GB concentrations.

$23 \% \mathrm{RH}$ and $13^{\circ} \mathrm{C}$ conditions. At high GB concentrations $\left(>1.0 \mathrm{mg} / \mathrm{m}^{3}\right)$, the sensor response was increasing linear with increasing GB concentration, which satisfies the law of the adsorption isotherm in solid (Fig. 8(a)). Also, when the gas was removed, the sensor response returned to $\sim 65 \%$ of its initial baseline value within $4 \mathrm{~min}$. The sensitivity in the GB concentration range of $2-16 \mathrm{mg} / \mathrm{m}^{3}$ was evaluated as $\sim 1,455.6 \mathrm{~Hz} / \mathrm{mg} / \mathrm{m}^{3}(\sim 274 \mathrm{~Hz} / \mathrm{ppm})$. This result is better than other reported values for similar devices. Rebiere et al. reported a GB sensor coated with fluoropolyol (FPOL) sensitive film incorporating $98 \mathrm{MHz}$ SAW delay line oscillator with a sensitivity of $\sim 250 \mathrm{~Hz} /$ ppm at the testing temperature of $45^{\circ} \mathrm{C}$ and humidity of $60 \% \mathrm{RH}$ [7]. In contrast, at low GB concentrations $\left(<1.0 \mathrm{mg} / \mathrm{m}^{3}\right)$, the sensor response was complicated, as shown in Fig. 8(b). In the initial part of the testing period, 
the sensor response increases with increasing concentration, however, the opposite phenomenon occurs upon further testing. A peak of the sensor response was observed at the GB concentration of $0.6 \mathrm{mg} / \mathrm{m}^{3}$, which is coincident with the adsorption isotherm in liquid. On the basis of the measured results, we suggest that at the tested temperature, viscous-liquid SXFA presents the properties of adsorption both in solid and in liquid.

\section{CONCLUSIONS}

A $300 \mathrm{MHz}$ SAW gas sensor based on a novel oscillator with high frequency stability was fabricated. A new SAW delay line on an ST-X quartz substrate with EWC/SPUDT (low loss with less than $12 \mathrm{~dB}$ ) and single-mode-selection capability was fabricated as the oscillator element. COM theory was used to predict the device performance prior to fabrication. The experimental results showed the short term frequency stability $(1 h)$ to be $\sim 0.07 \mathrm{ppm}(\sim 20 \mathrm{~Hz})$ in a laboratory environment with temperature control. SXFA polymer film was used to the sensitive material for GB detection. Superior sensitivity $\left(1,455 \mathrm{~Hz} / \mathrm{mg} / \mathrm{m}^{3}\right.$ in the concentration range of $2-14 \mathrm{mg} / \mathrm{m}^{3}$ ) and smaller threshold detection limit $\left(\ll 0.4 \mathrm{mg} / \mathrm{m}^{3}\right)$ were obtained.

\section{ACKNOWLEDGMENT}

This work was supported by the National High Technology Research and Development Program of China (Grant No. 2006AA06Z413) and the National Natural Science Foundation of China (Grant No. 10834010).

\section{REFERENCES}

[1] H. Wohltjen and R. Ressy, "Surface acoustic wave probe for chemical analysis, Parts I-III," Anal. Chem., 51, 1458-1475 (1979).

[2] Y. T. Shen, C. Y. Shen and L. Wu, "Surface plasmon resonance measurement of pyridoxal kinase-pyridoxamine binding on self-assembled monolayer," Sens. Actuators B, 85, 227-231 (2002).

[3] C. S. Hartmann and P. V. Wright, "Overview of design challenges for single phase unidirectional SAW filters," IEEE Ultrason. Symp. Proc., pp. 79-89 (1989).

[4] V. Plessky, "Coupling-of-modes Analysis of SAW devices," Int. J. High Speed Electron. Syst., 10, 31-33 (2000).

[5] P. V. Wright "Analysis and design of low-Loss SAW devices with internal Reflections using coupling-of-modes theory," IEEE Ultrason. Symp. Proc., 141-152 (1989).

[6] W. Wang, S. He and Y. Pang, "Enhanced sensitivity of SAW gas sensor based on high frequency stability oscillator," Smart Mater. Struct., 15, 1525-1530 (2006).

[7] D. Rebière. C. Dejous and J. Pistre, "Synthesis and evaluation of fluoropolyol isomers as SAW microsensor coatings: Role of humidity and temperature," Sens. Actuators B, 49, 139-145 (1998).

Shitang He received his B.S. degree from HuNan University, China, in 1982 and a M.S. degree from the Institute of Acoustics (IOA), Chinese Academy of Sciences (CAS) in 1987. He is currently working at IOA as a professor with research interests in SAW devices, sensors and MEMS.

Wen Wang received his B.S., and M.S. degrees from Central South University, China, in 1999 and 2002, respectively, and his Ph.D. degree from the IOA, CAS in 2005. His major research interests are chemical and physical applications of surface acoustic wave sensors, including gas sensors, RF micro-sensors and gyroscopes. He is currently working in IOA as an associate professor.

Shunzhou Li received his B.S. degree from Central South University, China, in 1982, and his M.S. degree from Beijing Normal University in 1988. His major research areas include hightemperature superconductor filters and microwave circuits. $\mathrm{He}$ is currently working at IOA as an associate professor.

Yong Pan received his B.S. degree from Zhengzhou University, China, in 1994, and his M.S. and Ph.D. degrees from the Research Institute of Chemical Defense in 2001 and 2004, respectively. He is currently working at the Research Institute of Chemical Defense as an associate professor with research interests in SAW chemical sensors.

Minghua Liu received his B.S. degree from HuNan University, China, in 2001. He is currently working at IOA, CAS, as an engineer interesting in SAW device techniques. 